28. Ota, K.: Kinetics of cellular proliferation in levkemia and cancer. Acta haemat. jap. 27, 693 -704 (1964).

29. Rogers, A. W.: Technies of autoradiography. AmsterdamLondon-New York: Elsevier Publ. Comp. 1967.

30. Pilgrim, C., Maurer, W.: Autoradiographische Bestimmung der DNS-Verdoppelungszeit versehiedener Zellarten von Maus und Ratte bei Doppelmarkierung mit $3 \mathrm{H}$ - und $14 \mathrm{C}$ Thymidin. Naturwissenschaften 49, 544-545 (1962).

31. - - Autoradiographische Untersuchung über die Konstanz der DNS-Verdoppelungsdauer bei Zellarten von Maus und Ratte durch Doppelmarkierung mit $3 \mathrm{H}$ - und 14C-Thymidin. Exp. Cell Res. 37, 183-199 (1965).

32. Quastler, $\mathbf{H}$. : The analysis of cell population kinetics. In: Lamerton and Fry's, Cell proliferation, p. 18-34. Oxford: Blackwell Sci. Publ. 1963.

33. - Sherman, F. G.: Cell population kinetics in the intestinal epithelium of the mouse. Exp. Cell Res. 17, 420-438 (1959).

34. Saunders, E. F., Lampkin, B. C., Mauer, A. M.: Variation of proliferative activity in leukemic cell populations of patients with acute leukemia. J. clin. Invest. 46, 1356$1363(1967)$.

35. Sherman, F. G., Quastler, H., Wimber, D. R.: Cell population kinetics in the ear epidermis of mice. Exp. Cell Res. $25,114-119$ (1961).

36. Sohutze, B.: Die Orthologie und Pathologie des Nukleinsäure- und Eiweißstoff wechsels der Zelle im Autoradiogramm. In: Handbuch der allgemeinen Pathologie, Bd. II: Die Zelle, Teil 5: Stoffwechsel und Feinstruktur der Zelle, redig. v. F. Büchner, S. $466-670$. Berlin-HeidelbergNew York: Springer 1968.

37. - Oehlert, W.: Autoradiographic investigation of incorporation of $\mathrm{H} 3$-thymidine into cells of the rat and mouse. Science 131, $737-738$ (1960).
38. Stryckmans, P., Ramos, J., Fliedner, T. M., Cronkite, E. P.: An estimate of DNA synthesis time in white and red cell precursors in human beings. Blood 24, 851 (1964).

39. - Cronkite, E. P., Fache, J., Fliedner, T. M., Ramos, J.: Deoxyribonucleic acid synthesis time of erythropoietic and granulopoietic eells in human beings. Nature (Lond.) 211, $717-720(1966)$.

40. Tonna, E. A., Cronkite, E. P.: An autoradiographic study of periosteal cell proliferation with tritiated thymidine. Lab. Invest. 11, $455-462(1962)$.

41. Trepel, F., Rastetter, J., Theml, H., Stockhusen, O. Nukleinsäuresynthese und Zytostatikawirkung in patho. logischen Lymphknotenzellen. Med. Klin. 61, 618-622 (1966).

42. Tsirimbas, A. D. : Markierung von Lymphdrüsen mit radioaktivem Thymidin in vivo beim Menschen. (In Vorbereitung.)

43. Wegener, K., Hollweg, S., Mauer, W.: Autoradiographische Bestimmung der Dauer der DNS-Verdoppelung und der Generationszeit bei fetalen Zellen der Ratte. Naturwissenschaften 50, 738-739 (1963).

44. Wimber, D. E.: Methods for studying cell proliferation with emphasis on DNA labels. In: Lamerton and Fry's, Cell proliferation, p. $1-17$. Oxford: Blackwell Sci. Publ, 1963.

45. - Quastler, H.: A 14C- and $3 \mathrm{H}$-thymidine double labeling technique in the study of cell proliferation in tradescantia root tips. Exp. Cell Res. 30, $8-22$ (1963).

Dr. A. D. Tsirimbas

Dr. P. Dörmer

Institut für Hämatologie

D-8000 München, Ziemssenstr. 1a

\title{
Eine verbesserte fluorimetrische Cortisolbestimmung im Serum: Diagnostische Bedeutung und therapeutische Folgerungen bei NNR-Insuffizienz
}

\author{
F. Kuvge, A. C. Gerb, N. Boss, R. Fahlbusoh and P. C. Soriba* \\ II. Medizinische Klinik (Komm. Direktor: Prof. Dr. M. M. Forell) und Neurochirurgische Klinik (Direktor: \\ Prof. Dr. F. Marguth) der Universität München
}

\section{Einleilung}

Die bisher bei uns übliche Bestimmung der sog. 11-Hydroxy-Corticosteroide $[4,6,9-12,14,20]$ ist methodisch unbefriedigend. Sie ist durch hohe unspezifische Basisfluorescenz, Störanfälligkeit und mangelnde Präzision belastet. Ausgehend von Untersuchungen über die Maxima der Absorption und Emission bei der fluorimetrischen Cortisolbestimmung $[5,19]$ wurden die technischen Voraussetzungen für eine exaktere fluorimetrische Serumcortisolbestimmung untersucht [8].

Nach Aufbau dieser Methode sollten folgende Fragestellungen geprüft werden:

1. Empfindlichkeit, Genauigkeit, Richtigkeit, Reproduzierbarkeit, Störfaktoren und Spezifität der Methode.

2. Normalbereiche für NNR-Gesunde (9 Uhr-Nüchterneortisol und ACTH-Belastung).

3. 24 Std-Rhythmus der Serumcortisolspiegel bei NNR-Gesunden oder bei Cortisolsubstitution von NNR-Insuffizienten; Verbesserung der Tagesverteilung der Cortisolsubstitution.

4. Kinetik des Absinkens der Serumcortisolspiegel bei Dexamethasonsuppression oder -substitution.

5. Diagnostische Wertigkeit der Methode und der i.v. ACTH-Belastung bei primärer und sekundärer NNR-Insuffizienz und beim Cushing-Syndrom.

* Mit Unterstützung der Deutschen Forschungsgemeinschaft (SFB 51).

\section{Methoden}

\section{Fluorimetrische Cortisolbestimmung}

a) Reagentien. Hydrocortison reinst (Merck 8963), Äthanol abs. p.A. (Merek 972), Sehwefelsäure 95-97\% p.A. (Merck 731), Dichlormethan p.A. (Merck 6050).

b) Blutentnahme. Die Blutentnahmen erfolgten um $9 \mathrm{Uhr}$ oder zu den angegebenen Zeiten aus einer Armvene. Das Blut wurde in Dichromatsehwefelsäure - gereinigten Glasröhrchen oder in Plastik-Einmalröhrchen aufgefangen. Nach Zentrifugation $(10 \mathrm{~min}, 1500 \mathrm{~g})$ wurde das Serum gleich oder nach Auftauen (Lagerung bei $-20^{\circ} \mathrm{C}$ ) verwendet.

c) Fluorescenzreagens. Konzentrierte Schwefelsäure und Äthanol abs. werden bei $+4^{\circ} \mathrm{C}$ gelagert. Zu $150 \mathrm{ml}$ Äthanol abs. $\left(+4^{\circ} \mathrm{C}\right)$ werden $350 \mathrm{ml}$ konz. $\mathrm{H}_{2} \mathrm{SO}_{4}\left(+4^{\circ} \mathrm{C}\right)$ innerhalb von ca. $2 \mathrm{Std}$ über einen Tropftrichter gegeben (Eisbad, Magnetrührer). Das frisch angesetzte Fluorescenzreagens muß nach vollständiger $\mathrm{H}_{2} \mathrm{SO}_{4}$-Zugabe noch 2 Std weiter und auch während der Entnahme der $4 \mathrm{ml}$ Proben, die zum Dichlormethanextrakt zugesetzt werden, im Eisbad gerïhrt werden, um konstante Leerwerte zu erhalten.

d) Extralktion. $2 \mathrm{ml}$ Serum werden mit $10 \mathrm{ml}$ Dichlormethan in $20 \mathrm{ml}$ Schliffstopfengläsern mit einem Rotationsmischer, dessen Achse zur Horizontalen eimen Winkel von $20^{\circ}$ bildet, extrahiert ( $20 \mathrm{~min}, 40 \mathrm{UpM}$, Zimmertemperatur). Nach vorsichtigem Absaugen der wäßrigen Phase wird der Dichlormethanextrakt mit $2 \mathrm{ml} \quad 0,1 \mathrm{~N} \mathrm{NaOH}$ gewaschen (Whirlmix, $10 \mathrm{sec}$ ) und mittels vorsichtigem Absaugen und Dekantieren von der wäßrigen Phase getrennt. $Z u \quad 7,5 \mathrm{ml}$ Dichlormethanextrakt kommen in $20 \mathrm{~cm}$ langen graduierten Schliffgläsern $4 \mathrm{ml}$ eiskalten Fluorescenzreagenses (s.o.); $30 \mathrm{sec}$ Mischen (Whirlmix). Nach Entmischung der Phasen (ca. $10 \mathrm{~min}$ Stehen) wird der Dichlormethanüberstand abgesaugt, wobei die Vermeidung des Ansaugens von Luft entscheidend ist [8]. Dazu wird eine $0,2 \mathrm{ml}$ Pipette in die Dichlormethanphase bis kurz über das fluorescenzreagens getaucht. 


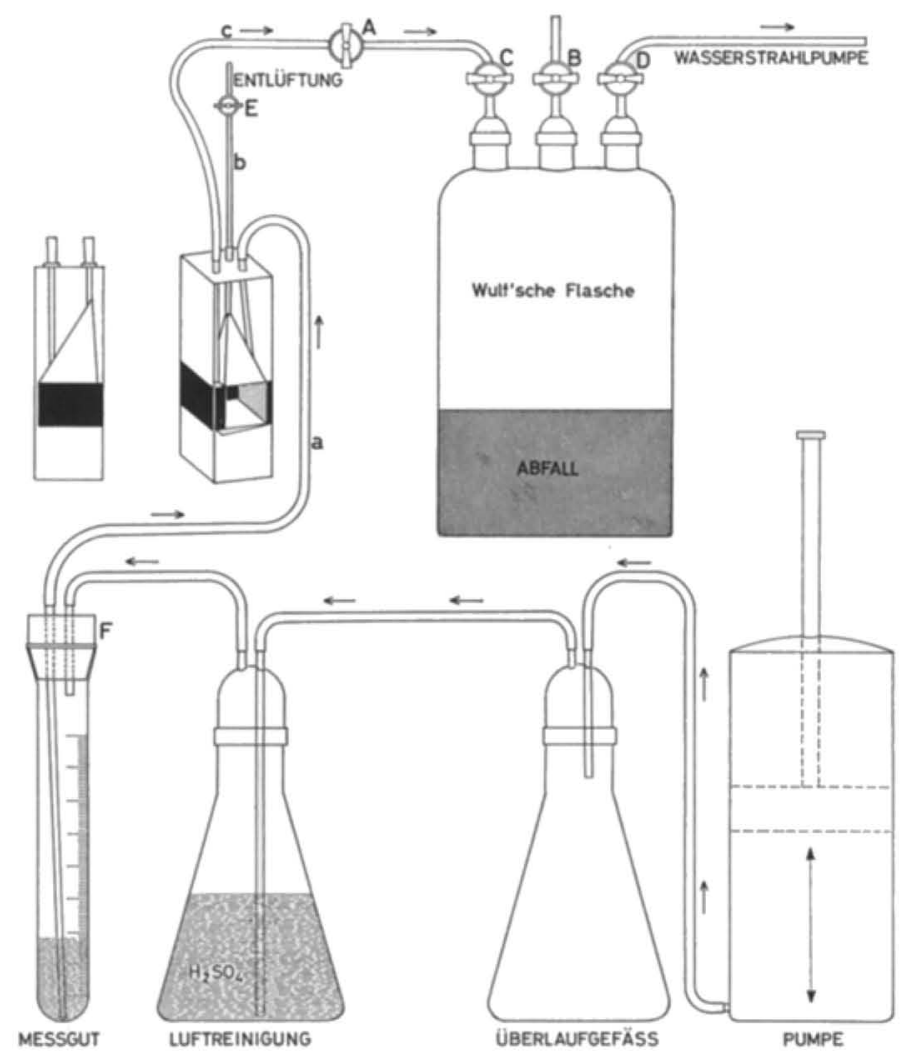

Abb. 1. Mikroabsaugküvette, Füllungsw und Entleerungsvorgang. - Mikroabsaugküvette: Drei Seitenwände der Küvette sind aus Schwarzglas, Schichtdicke $=1 \mathrm{~cm}$, Füllvolumen $=1,2 \mathrm{ml}$, Einfüllstutzenende im schrägen unteren Drittel des Kamins, Entlüftungsstutzen an der Spitze und Absaugstutzen am tiefsten Punkt des Füllraumes. Die Küvette wurde in Abänderung der Küvette Nr. 185 der Fa. Hellma, Müllheim/Baden, gebaut. - Füllung: Hahn $A$ und $D$ zu, Hahn $C$ und $B$ auf. Wenn der Kunststoffstopfen $F$ luftdicht auf das graduierte Schliffglas $(20 \mathrm{ml})$ gesetzt wird, drückt getrocknete und gereinigte Luft das Meßgut durch Sehlauch $a$ in die Küvette, wobei die Luft über den geöffneten Hahn $E$ entweichen kann. Wenn die Küvette gefüllt ist, wird Hahn $E$ geschlossen und Hahn $A$ geöffnet, bis die Flüssigkeitssäule Hahn $A$ erreicht. Zugleich wird die Leistung der Aquariumspumpe gedrosselt, damit der Druck in der Küvette nicht zu hoch wird. Die Messung kann bereits beginnen, wenn der Hahn $E$ geschlossen ist. - Entleerung: Während der Messung wird die Wulfsche Flasche evakniert (Hahn $C$ und $B$ geschlossen und $D$ geöffnet). Nach der Messung (15 sec) wird bei geschlossenem Lichtweg die Küvette durch Öffnung der Hähne $A$ und $C$ leergesaugt. Nach Entleerung des Schlauches $c$ wird auch Hahn $E$ geöffnet und schließlich der Stopfen $F$ gelockert. Entscheidend ist bei diesem Füllvorgang die Vermeidung des Entstehens von feinsten Bläschen in dem mit Dichlormethan gesättigten Fluorescenzgemisch, welche durch Reflektion in der Küvette die Messung verfälschen. Das geschieht durch Füllung der Küvette mittels einer Pumpe gegen die Summe von atmosphärischem Druck und Strömungswiderstand in der Entlüftungscapillare

Gerade vor dem Erreichen der Grenzschicht wird die Pipettenspitze schnell in das Fluorescenzreagens getaucht und wieder angehoben, so daß der Dichlormethanrest und die obersten $0,2-0,3 \mathrm{ml}$ des Fluorescenzreagenses wegen der höheren Viscosität des Fluorescenzreagenses Iangsam abgesaugt werden.

e) Fluorimetrie. $79 \mathrm{~min}$ nach dem Mischen des Fluorescenzreagenses und des Dichlormethanextraktes wird die Mikroabsaugküvette (Abb. 1) nach dem Schema ( $\mathrm{Ab}$. 1) gefüllt; in der 80 . min wird gemessen. Beim registrierenden Spektral. fluorimeter Beckmann mit Xenon-Hochdrucklampe (XE 1045) ist der erste Monochromator (GM 1139) auf $464 \mathrm{~nm}$ eingestellt (Spaltbreite entspricht $20 \mathrm{~nm}$ ); die Emission wird bei $522 \mathrm{~nm}$ (Spaltbreite entspricht $5 \mathrm{~nm}$ ) im Auflichtverfahren gemessen. Das Gerät ist so geeicht, daß $0-30 \mu \mathrm{g}$ Cortisol pro $100 \mathrm{ml}$ Eichlösung 100 Skalenteilen (SKT) entsprechen. Diese Spreizung läßt sich bei Bedarf ohne weiteres auf das 3fache erhöhen. Cortisolwerte über $30 \mu \mathrm{g} / 100 \mathrm{ml}$ sind durch Stufenschaltung ablesbar. Die Überprüfung der Richtigkeit der $A b$ wägungen und Verdünnungen für Eichkurven ergab bei einem Arbeitsstandard von $10 \mu \mathrm{g}$ Cortisol pro $100 \mathrm{ml}$ bei 10maligem $\mathrm{Ab}$ wiegen and Verdünnen einen Mittelwert von $\bar{x} \pm s=10,09 \pm$ $0,42 \mu \mathrm{g} / 100 \mathrm{ml}, \mathrm{VK}=4,2 \%$. Das mittlere Körpergewicht der Normalpersonen $(\bar{x} \pm s)$ betrug $103,8 \pm 12,7 \%$ des Durchschnittsgewichtes (nach Geigy-Tabellen). Eine Korrelation zwischen Körpergewicht dieser Personen und ihren $9 \mathrm{Uhr}$ Nüchterncortisolwerte bestand nicht $(r=0,2$; nicht signifi. kant).

\section{Intravenöse ACTH-Belastung}

Je $0,25 \mathrm{mg}$ Synacthen ${ }^{\circledR}$, frisch gelöst in $500 \mathrm{ml} 5 \%$ Laevu. loselösung, wurden an zwei aufeinander folgenden Tagen von $9 \mathrm{Uhr}$ bis $13 \mathrm{Uhr}$ infundiert. Die Seren A, B, C und D wurden durch Blutentnahme vor und nach ACTH-Gabe, also um 9 und um $13 \mathrm{Uhr}$ jeweils an beiden Tagen gewonnen (Abb. $3 \mathrm{a}, \mathrm{b}$ ).

\section{Messung des Serumcortisols im 24 Std-Rhythmus mit und ohne Cortisolsubstitution}

NNR-insuffiziente Patienten wurden um 7 Uhr mit $15 \mathrm{mg}$ Cortisol, uml3 Uhr mit $5 \mathrm{mg}$ und um $19 \mathrm{Uhr}$ mit $10 \mathrm{mg}$ oral substituiert. Um $6,9,12,18,24$ und $6 \mathrm{Uhr}$ wurde bei diesen Patienten und ebenso bei einem Kollektiv Stoffwechselgesunder Blut zur Cortisolbestimmung entnommen (Abb.4).

\section{Bestimmungen an ,Cortisol-freiem" Serum}

(Dexamethason-Substitution bei NNR-Insuffizienz)

NNR-insuffiziente Patienten erhielten zuletzt um $7 \mathrm{Uhr}$ oral $15 \mathrm{mg}$ Cortisol; am gleichen Abend sowie am Morgen und Abend der folgenden Tage wurden sie mit jeweils $0,25 \mathrm{mg}$ Dexamethason substituiert, welches durch die fluorimetrische Bestimmung nicht erfaBt wird [12]. Am ersten Tag wurde um ca. 9, 12 und $18 \mathrm{Uhr}$ und an den folgenden 2-3 Tagen jeweils gegen 9 und $18 \mathrm{Uhr}$ Blut entnommen $(\mathrm{Abb} .5,6)$. 


\section{Ergebnisse}

1. Zur fluorimetrischen Cortisolbestimmung

Die llethode erlaubt die Bestimmung von weniger als 1 : $\ell$ Cortisol in $100 \mathrm{ml}$ Eichlösung (Empfindlichkeit: $\bar{x} \pm s=1,0 \pm 0,3 \mu \mathrm{g} / 100 \mathrm{ml}, N=20$, Variations$k$ effizient VK $=30 \%$ ). Sie ist durch eine hohe Gerauigkeit an einem Tag (Eichlösung von $10 \mu \mathrm{g} / 100 \mathrm{ml}$ : $\bar{x} \pm s=10,7 \pm 0,5 \mu \mathrm{g} / 100 \mathrm{ml}, \quad N=20, \mathrm{VK}=4,7 \% ;$ Kontrollserum: $\mathrm{VK}=3,8 \%$ ) ausgezeichnete Richtigkeit (Wiederfindeversuche für Cortisol \pm Serum, Korrelation $r=0,999$; Regression $b=0,964)$ und Reproduzierbarkeit von Tag zu Tag (Kontrollserum: $\mathrm{VK}=6-7 \%$ ) gekennzeichnet. Dì Spezifität der Methode zeigte sich an den niedrigen Serumcortisolwerten bei Dexamethasonsuppression und -substitution (Abb. 5, 6). Serum- und Plasmawerte sind gleich $(r=0,995$; Regression $b=0,999)$. Eine geübte Kraft kann mit dieser Methode 34 Seren in 8 Std untersuchen (Doppelbestimmungen).

\section{Normalbereiche}

Soldaten $(N=37)$, Personal $(N=22)$ und stationäre nebennierengesunde Patienten $(N=43)$ zeigten zusammen eine logarithmische Verteilung der $9 \mathrm{Uhr}$ Nüchterncortisolwerte im Serum: Normalbereich 9,7 bis $32,0 \mu \mathrm{g} / 100 \mathrm{ml}$ (Abb. 2). Auch bei i.v. ACTHBelastungen Gesunder sind die 9- und 13 Uhr-Werte des Serumcortisols logarithmisch verteilt (Abb. 3).

\section{Intravenöse ACTH-Belastung}

Bei der Durchführung von i.v. ACTH-Belastungen zeigte sich ein deutlich unterschiedliches Verhalten bei Normalpersonen sowie primär und sekundär NNRinsuffizienten Patienten (Abb. 3a). Addison-Patienten $(N=5)$ waren bereits durch Bestimmung des 9 UhrNüchterneortisols zu diagnostizieren und wiesen keine Reaktion auf die ACTH-Infusion auf; die Mittelwerte für die Seren A, B, C und D sind hochsignifikant $(p<0,005)$ von den Vergleichswerten für Normalpersonen unterschieden (Abb. 3a). - Bei Patienten, die nach Operation eines Hypophysentumors untersucht wurden $(N=30)$, waren die entsprechenden Werte beim ACTH-Test im Vergleich zu Normalpersonen signifikant erniedrigt (Abb. 3a). Sie lagen aber höher als bei primärer NNR-Insuffizienz.

Entscheidend für die diagnostische Brauchbarkeit der Methode ist jedoch nicht die Tatsache, daß die Werte von NNR-insuffizienten Patienten und an Hypophysentumoren Operierten im Mittel signifikant unter den Normalbereichen liegen. Viel wichtiger ist die Frage, wieviele Werte der Patienten dieser inhomogenen Kollektive außerhalb der Normalbereiche liegen (Abb. 3a). Alle Werte der 5 Addisonpatienten lagen sicher unterhalb der Normalbereiche. Von den hypophysenoperierten Patienten lagen bei A: 22 von 30 , bei B : 23 von 30 , bei C: 11 von 29 und bei D: 19 von 29 Werten unterhalb des Normalbereiches. Eine sekundäre NNR-Insuffizienz ließ sich also nach den Werten des ersten Tages bei ca. $75 \%$ der wegen eines Hypophysentumors Operierten nachweisen [9, 18]. Die 9 Uhr-Nüchternwerte von 5 Patienten mit einem Cushing-Syndrom (Abb. $3 \mathrm{~b}$ ) liegen ebenso wie die Werte beim ACTH-Belastungstest oberhalb des Normalbereiches NNR-Gesunder.

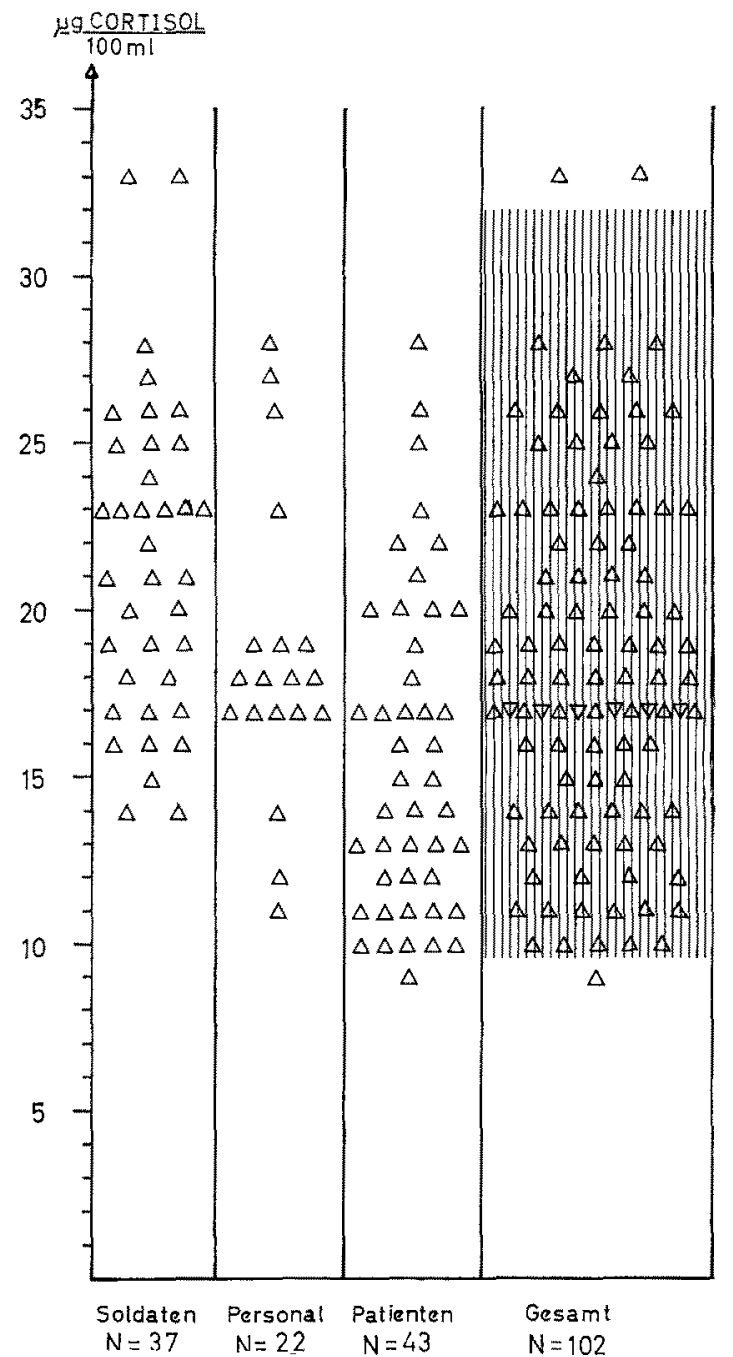

Abb. 2. 9 Uhr-Nüchterncortisolwerte von Normalpersonen. Der Normalbereich (logarithmische Verteilung, $\tilde{x} \pm 2 s$ ) ist schraffiert, der Mittelwert ist $17,6 \mu \mathrm{g} \%$ [8]

\section{24 Std-Rhythmus des Serumcortisols bei Normal- personen und bei Cortisolsubstitution von NNR-Insuffizienten}

Um die Effektivität unseres Substitutionsschemas für NNR-insuffiziente Patienten [15a, 17] zu prüfen, untersuchten wir die Serumcortisolspiegel im 24 StdRhythmus (s. Methoden). Dabei zeigte sich, daß bei Addison-Patienten $(N=6)$ und bei einem totaladrenalektomierten Patienten die Serumcortisolspiegel teilweise unter den Mittelwerten des Tagesprofils von NNR-Gesunden liegen. Dies ist besonders in den frühen Morgenstunden der Fall (Abb. 4).

\section{Bestimmungen an „Cortisol-freiem" Serum}

(Dexamethason-Substitution bei NNR-Insuffizienz)

Messungen sehr niedriger Cortisolspiegel sind wichtig für die Diagnostik der NNR-Insuffizienz und für Aussagen über die Spezifität dieser Methode. Die Cortisolspiegel sanken unter der angegebenen Dexamethasonsubstitution (s. Methoden) in der angegebenen Zeit bei den Addison-Patienten $(N=6)$ auf 2 bis $4 \mu \mathrm{g} / 100 \mathrm{ml}$ und bei den Totaladrenalektomierten $(N=3)$ auf $1-2 \mu \mathrm{g} / 100 \mathrm{ml} \mathrm{ab}(\mathrm{Abb} .5,6)$. 

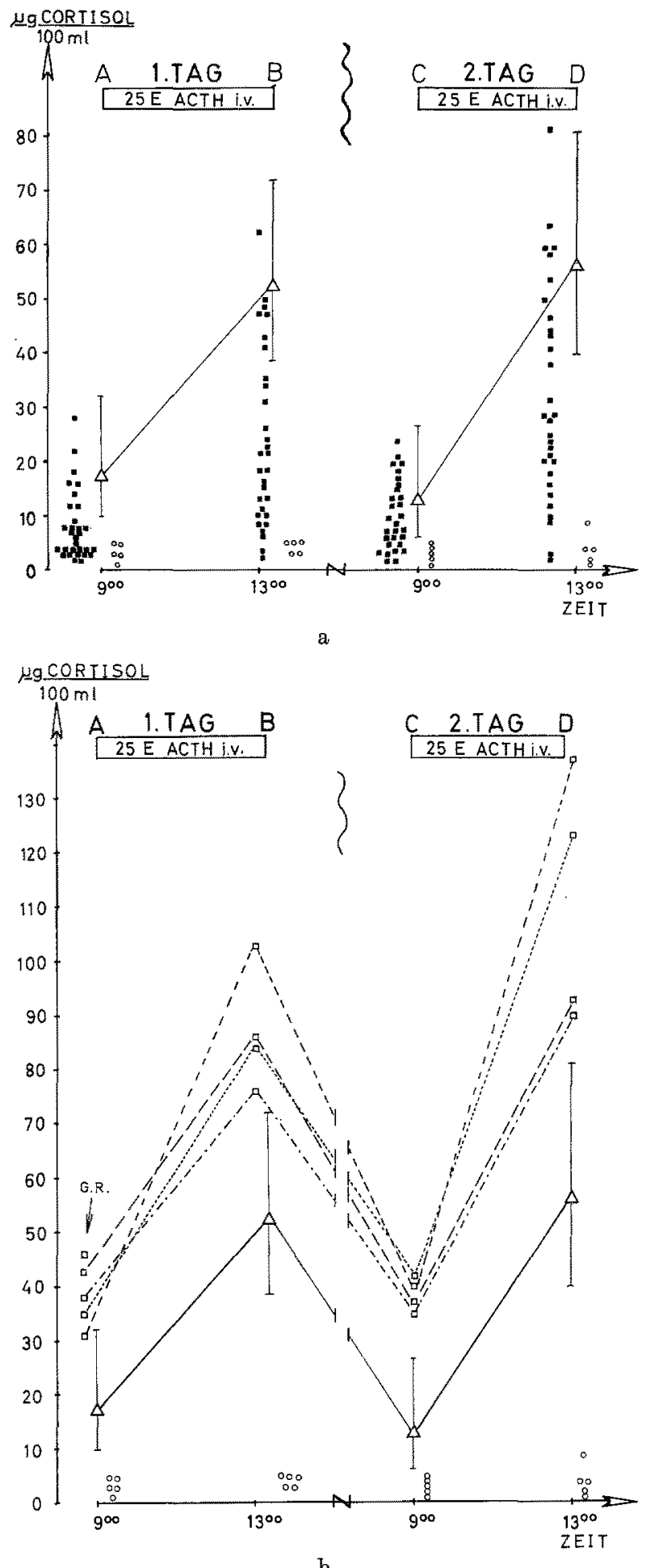

Abb. 3a a. b. ACTH-Belastungen, Durchführung s. Methoden. Stoffwechselgesunde $(\Delta-\Delta)$, Zustand nach Operation eines Hypophysentumors ( $\mathbf{0})$, Morbus Addison (O), CushingSyndrom ( $\square-\square)$. Bei der Patientin M. L. handelte es sich um ein NNR-Adenom. Die Patientin G. R. hatte ein NNR-Carcinom, die Patientinnen R.Z., G. A. und M. H. hatten eine bilaterale NNR-Hyperplasie.

a $\Delta$ Stoffwechselgesunde, $\bar{x} \pm 2$ s (geom.),$N=23$. - Hypophysektomierte, $N=30$. $\circ$ Addison-Patienten, $N=\breve{5}$.

b $\square$ Cushing-Patienten, $N=$ 5. M. H. $\square--\square$, G.A. $\square-\square-\square$, R.Z. $\square \cdots \cdots \cdot \square$, M. L. $\square-\cdots \cdot \square$, G.R. $\square$ (Einzelwert). $\triangle$ Stoffwechselgesunde, $\bar{x} \pm 2$ s (geom.), $N=23$. Addison-Patienten $N=5$

\section{Diskussion}

Das praktische Kriterium dieser Methode zur Cortisolbestimmung ist die klinische Brauchbarkeit.

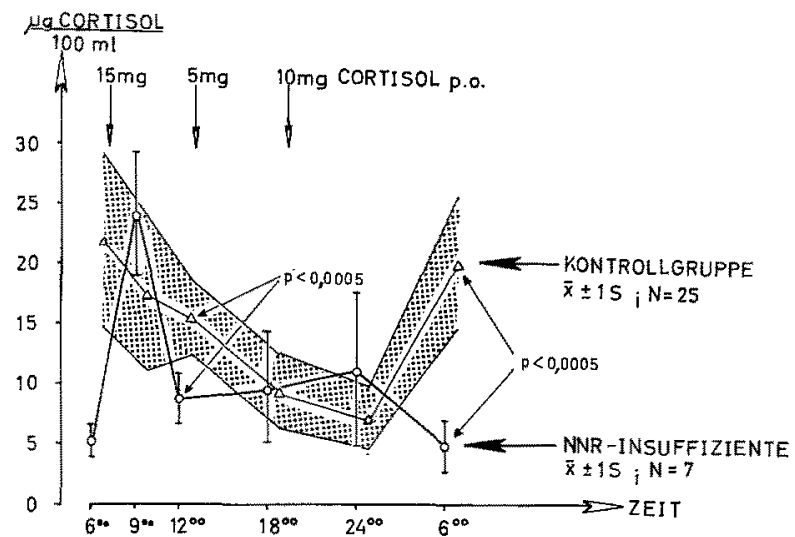

Abb. 4. Serumcortisolspiegel im 24 Std-Rhythmus $(\tilde{x}+s)$. Cortisolsubstitution s. Methoden. Stoffwechselgesunde $(\Delta-\Omega)$ $-\triangle)$ and Patienten unter Cortisolsubstitution $(0-0)$ : Morbus Addison $(N=6)$, Totaladrenalektomiert $(N=1)$

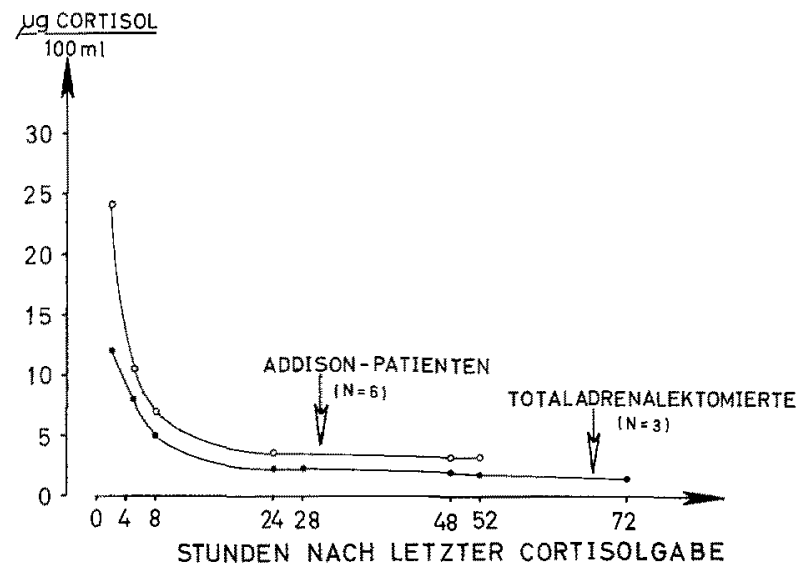

Abb. 5. Absinken von Cortisolspiegeln unter Dexamethasonsubstitution von Patienten mit Morbus Addison $(0-0)$, bzw. nach totaler Adrenalektomie (-C), Mittelwerte

Insbesondere muß hierbei an exakt reproduzierbare Meßwerte für NNR-insuffiziente Zustände gedacht werden. Aber gerade die unscharfe Trennung solcher Zustände gegenüber normalen Meßwerten belastet bisher angewandte Methoden $[4,6,7,9-14,20]$.

$\mathrm{Zu}$ einer technisch befriedigenden Lösung gelangten wir u.a. durch den Einbau einer speziellen Mikroabsaugküvette, angeschlossen an ein Füllsystem aus Teflonschläuchen mit einer regelbaren Pumpe (Abb. 1). Hierdurch konnte die Aufgabe gelöst werden, diese Küvette blasenfrei zu füllen, da feinste reflektierende Bläschen die Messung dureh Störstrahlung beeinträchtigen. Das Spektralfluorimeter ermöglichte eine Erhöhung der Spezifität der Anregung und Emission bei genügend hoher Lichtintensität, wodurch die Nachteile der sekundären Verstärkung der Emission nicht mehr ins Gewicht fielen. Durch die Verlegung des Meßzeitpunktes wurde erreicht, daß die Fluorescenzintensität von Cortisol stabil war. Außerdem steigt dadurch die Spezifität der Methode, da unspezifische Fluorigene ihr Fluorescenzmaximum schnell erreichen, während die Cortisolfluorescenz bis zur 70.-80. min zunimmt $[6-8,20]$.

Mit diesen Verbesserungen ist die Methode so empfindlich, daß bereits mit der Bestimmung des 9 Uhr-Nüchterncortisols eindeutig zwischen normalen und primär und z.T. auch sekundär NNR-insuffizien- 


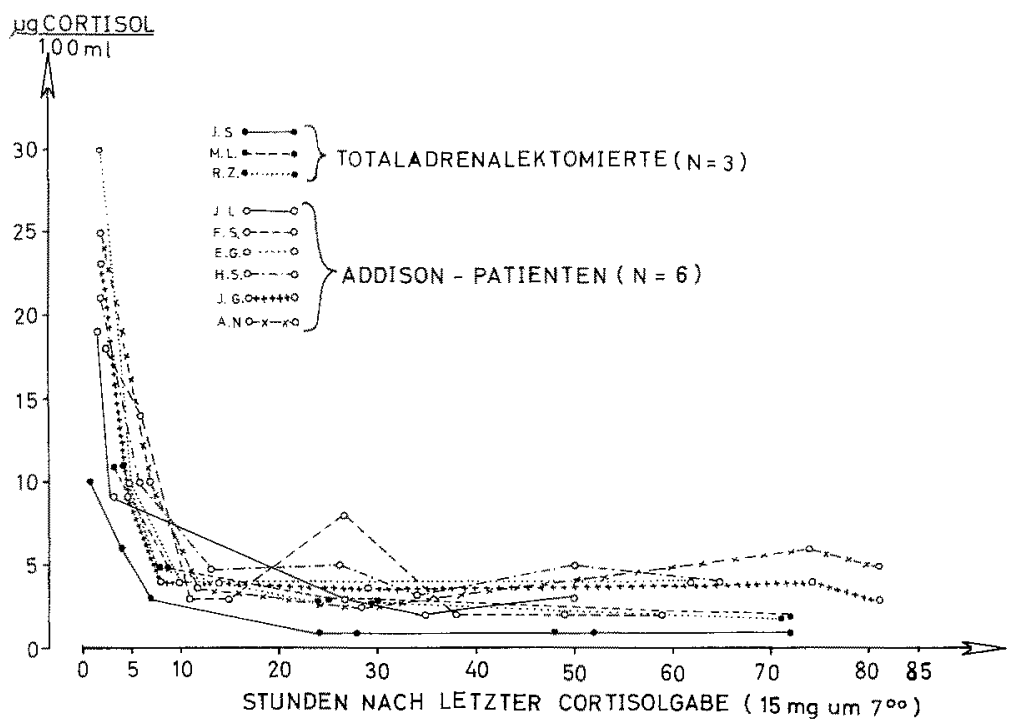

Abb. 6. Absinken von Cortisolspiegeln unter Dexamethasonsubstitution von Patienten mit Morbus Addison, bzw. nach Totaladrenalektomie. Dargestellt sind die Einzelverläufe von 9 Patienten

ten Patienten (Abb. 3a) unterschieden werden kann. Zur Unterscheidung zwischen einer inkompletten und kompletten NNR-Insuffizienz ist hingegen der ACTHTest immer noch erforderlich.

Zieht man die Spezifität der Methode in Betracht, so sollte man sich darüber im klaren sein, daß zu ihrer Prüfung grundsätzlich zwei Wege offenstehen, wobei theoretisch gesehen kein Weg zu einer vollständigen Verifikation unserer Hypothese führt, nämlich daß wir nur Cortisol (und Corticosteron ${ }^{1}$ ) bestimmen. Denn Millionen positiver Fälle genügen nicht, um ein solches statistisches Gesetz zu verifizieren; ein Gegenbeispiel genügt jedoch, um es zu falsifizieren [3].

Einerseits könnte man einen Negativkatalog aufstellen, indem wir möglichst viele Substanzen (Hormone, Medikamente) mit unserer Methode hinsichtlich der Störung der Cortisolbestimmung untersuchten. Hier muß man auf Störfaktoren, die die Fluorescenz erheblich im Sinne von erhöhten Werten beeinflussen, hinweisen. Es handelt sich um Silikonfette, Gummi sowie dessen Weichmacher und Aldactone $\mathbb{Q}[\mathbf{2}, 9,15$, 16].

Davon ausgehend, daß wir hauptsächlich Cortisol bestimmen, muß man andererseits erwarten, daß definierten klinischen Zustandsbildern ganz bestimmte Cortisolwerte zuzuordnen sind. Dies wurde durch folgende Versuchsanordnungen und deren Ergebnisse überprüft:

1. Es wurde ein Normalbereich NNR-gesunder Personen erstellt (Abb. 2).

2. Weiterhin wurde die Hypothalamus-Hypophysen-NNR-Achse nebennierenrindengesunder Personen $(N=17)$ mit Dexamethason (4mal $2 \mathrm{mg} / \mathrm{Tag}$ ) supprimiert. Am 1. Tag der Suppression konnten wir gegenüber dem Normalbereich schon stark erniedrigte Spiegel messen, die am 2. Tag aber noch geringfügig abfielen. Am 2. Tag fanden sich Cortisolspiegel von 2,2-3,7 $\mu \mathrm{g} / 100 \mathrm{ml}$ (Extremwerte, Gerb [8]).

3. Es wurden Dauer und AusmaB der Suppression der Hypothalamus-HVL-NNR-Achse durch einmalige

1 Corticosteron ergibt bei diesem Verfahren eine 3,2fach stärkere Fluorescenzintensitäit als Cortisol (0. A. Müller). orale, verschieden hohe Corticoiddosen beim Menschen quantitativ erfaßt and verglichen $[1]$.

4. Unter Dexamethasonsubstitution (2mal 0,25 mg/ Tag) gelang der Nachweis niedrigster Cortisolspiegel bei Addison- und adrenalektomierten Patienten ( $\mathrm{Abb}$. 5, 6). Im abfallenden Kurvenverlauf (Abb.5) zeigt sich am 3. Tag noch keine eindeutige Plateaubildung. Damit ist wahrscheinlich, daß die gemessenen niedrigen Werte zumindest z.T. noch Cortisol entsprechen und nicht ohne weiteres als unspezifische Basisfluorescenz abgezogen werden dürfen.

Zur Substitutionstherapie NNR-insuffizienter $\mathrm{Pa}$ tienten (Abb. 4) sei noch bemerkt, daß auch bei der bis jetzt bei uns üblichen Dosisverteilung [15a] noch Phasen eines Cortisolmangels vorhanden sind. Dies ist besonders in den frühen Morgenstunden und mittags der Fall. Diese ,Cortisoldurststrecken" [17] könnten so abgefangen werden, daß die Gesamt-Dosis noch besser verteilt würde:

Beim Aufstehen (ca.6 Uhr) $10 \mathrm{mg}$, um $10 \mathrm{Uhr}$ und 14 Uhr jeweils $5 \mathrm{mg}$, gegen $20 \mathrm{Uhr}$ nochmals $10 \mathrm{mg}$ Cortisol (Hydrocortison $(\mathrm{B})$ Hoechst). Zusätzlich kann man bei Hypotonie bzw. Mineralocorticoidmangel morgens ca. $0,1 \mathrm{mg}$ 9- $\alpha$-Fluohydrocortison (Fludrocortison = Florinef( Squibb) verabreichen .

Zusammenfassung. Die fluorimetrische Methode zur Bestimmung von Serumcortisol wurde durch blasenfreie Füllung einer Spezialküvette mittels Pumpvorrichtung, durch Benützung eines Spectralfluorimeters, sowie Verlegung des Meßzeitpunktes ( $80 \mathrm{~min}$ ) und durch optimale Anregung (464 nm) und Emissionsmessung $(522 \mathrm{~nm})$ verbessert. Empfindlichkeit $(<1 \mu \mathrm{g}$ Cortisol $/ 100 \mathrm{ml}$ ), Richtigkeit, Genauigkeit, Reproduzierbarkeit von Tag zu Tag (VK $=6-7 \%$ ) und Störfaktoren der Methode werden angegeben.

Mit dieser Methode wurden Normalbereiche für die 9 Uhr-Nüchterncortisolwerte und die i.v. ACTH: Belastung ermittelt.

Bei NNR-insuffizienten Patienten (M. Addison; Zustand nach Operation eines Hypophysentumors; total Adrenalektomierte) wurden i.v. ACTH-Belastungen durchgeführt, wobei sich bereits beim 9 Uhr- 
Nüchterncortisolwert eine diagnostisch gut brauchbare Trennung gegenüber dem Normalbereich ergab. Unter Dexamethasonsubstitution wurden bei NNR-Insuffizienz sehr niedrige Cortisolspiegel gemessen, was für die Spezifität der Methode spricht.

Die Bestimmung des 24 Std-Rhythmus der Cortisolwerte bei NNR-Insuffizienten zeigte, daß besonders in den frühen Morgenstunden im Vergleich zu Normalpersonen erniedrigte Cortisolspiegel bestehen. Daraus wird ein besserer Verteilungsvorschlag für die Cortisolsubstitution abgeleitet.

Summary. The fluorimetric determination of serum cortisol was improved

1. using a pumpdevice to fill a special microcu. vette avoiding the development of small bubbles,

2. using a recording spectrofluorometer with optimal absorption (464 nm) and emission (522 nm), and

3. allowing for maximal fluorescence of cortisol (80 $\mathrm{min})$.

Sensitivity $(<1 \mu \mathrm{g}$ cortisol/100 ml), accuracy, precision and specificity of the method are reported. Normal values of $9.00 \mathrm{a} . \mathrm{m}$. serum cortisol (9.7-32.0 $\mu \mathrm{g} / 100 \mathrm{ml}$ ) and of values before and after ACTH infusion tests were determined.

For adrenal insufficiency (Addisons disease, total adrenalectomy, or after hypophysectomy) the 9.00 a.m. values of serum cortisol were generally satisfactory for diagnosis. In partial adrenal insufficiency ACTH infusion tests had to be performed.

Very low levels of serum cortisol $(2-4 \mu \mathrm{g} / 100 \mathrm{ml})$ were obtained, when patients with adrenal insufficiency were substituted with dexamethasone for three days, proving the specificity of the method.

Determination of circadian rhythms of serum cortisol in patients with adrenal insufficiency on cortisol substitutive therapy in divided doses demonstrated cortisol levels far below the normal values during the early morning hours. This situation should be improved by dividing the cortisol dose as follows: 6 a.m.: $10 \mathrm{mg}, 10$ a.m.: $5 \mathrm{mg}, 2$ p.m.: $5 \mathrm{mg}$ and 8 p.m. or later: $10 \mathrm{mg}$ cortisol.

Fräulein R. Fröhlich danken wir für ihre ausgezeichnete technische Assistenz.

\section{Literatur}

1. Boss, N., Kluge, F., Gerb, A., Hoffmann, H., Seriba, P. C.: Suppressionwirkung von Corticoiden beim Menschen. Symp. dtsch. Ges. Endokrin. 16, im Druck (1970).

2. - Diss. Univ. München (in Vorbereitung).

3. Carnap, R.: Einführung in die Philosophie der Natur. wissenschaft, S. 29. München: Nymphenburger Verlagshandlung 1969.

4. Cope, C.L.: The adrenal cortex in internal medioine. Brit. med. J. $\mathbf{1 9 6 6 \mathrm { II } , 8 4 7 .}$
5. Daly, J. R., Spencer-Peet, J.: Fluorimetric determination of adrenal corticosteroids: Observations on interfering fluorogens in human plasma. J. Endocr. 30, 255 (1964).

6. Dörner, G., StahI, F.: Die diagnostische Bedeutung einer einfachen fluorimetrischen Routinemethode zur Beurteilung der Nebennierenrindenfunktion. Dtsch. med. Wschr. 90, $1917(1965)$.

7. - Eine einfache fluorometrische Methode zur Routinebestimmung von freiem Cortisol und Corticosteron im Harn. Aota biol. med. germ. 12, 606 (1964).

8. Gerb, A.: Eine verbesserte fluorimetrische Methode zur Bestimmung von „Cortisolspiegeln" im Serum. Diss. Univ. München 1969.

9. Hochheuser, W., Marguth, F., Müller-Bardorff, M., Schwarz, K., Scriba, P. C., Thiele, H.: Diagnostische Bedeutung der Proteinbindung von Plasmacortisol, bestimmt durch Dextrangelfiltration. Klin. Wschr. 47, 300 (1969).

10. - Müller-Bardorff, M., Scriba, P. C., Schwarz, K. : Fluorimetrische Bestimmung der 11-Hydroxycorticosteroide im Plasma unter der Therapie mit Corticoiden. Symp. dtseh. Ges. Endokrin. 12, 255 (1967).

11. - - Schwarz, K., Scriba, P. C.: Fluorimetrische Bestimmung der 11-Hydroxycorticosteroide im Plasma bei Hyperthyreose und Nebennierenrindeninsuffizienz. Symp. dtsch. Ges. Endokrin. 18, 298 (1968).

12. Mattingly, D.: A simple fluorimetric method for the estimation of free 11-hydroxycorticosteroids in human plasma. J. clin. Path. 15, 374 (1962)

13. - Dennis, P. M., Pearson, J., Cope, C. L.: Rapid screening test for adrenal cortical function. Lancet $1964 \mathrm{I}, 1046$.

14. Moor, P. de, Steeno, O.: Comparison of three techniques for the fluorimetric determination of plasma corticosteroids. J. Endoer. 28, 59 (1963).

15. - - Brosenes, J., Hendrix, H.: Data on transcortin activity in human plasma as studied by gel filtration. $J$. clin. Endocr. 26, 71 (1966).

15a. Schwarz, K., Dieterle, P., Hochheuser, W., Kollmannsberger, A., Müller-Bardorff, M., Scriba, P. C.: Zur Klinik der Nebennierenrindeninsuffizienz. Med. Klin. 62, 551 (1967).

16. Scriba, P. C., Werder, K. v., Richter, J., Schwarz, K. Ein Beitrag zur klimischen Diagnostik des ektopischen ACTH.-Syndroms. Klin. Wschr. 46, 49 (1968).

17. - Zur Pathophysiologie und Therapie der NNR-Insuffizienz. Schriftenreihe der Bayer. Landesärztekammer 17, $59(1969)$.

18. - Postoperative Diagnostik und Substitutionstherapie bei Hypophysentumoren (Referat). Symp. dtsch. Ges. Endokrin. 15, 274 (1969).

19. Spencer-Peet, J., Daly, J. R., Smith, V.: A simple method for improving the specificity of the fluorimetric determination of adrenal corticosteroids in human plasma. $J$. Endocr, 31, 235 (1965).

20. Stahl, F., Dörner, G.: The specifity of a simple routine method for the determination of unconjugated cortisol and corticosterone (11-OHCS) in urine. Acta endocr. (Kbh.), Suppl. 100, 67 (1965).

PD Dr. med. P. C. Scriba.

Dr. med. F. Kluge cand. med, A. C. Gerb cand, med. N. Boss

II. Med. Klinik der Universität D.8000 München. 15, Ziemssenstrasse 1

Dr. med. R. Fahlbusch

Neurochirurgische Klinik

der Universität

D-8000 München 15, Beethovenplatz 3 